\title{
VISION IN NIGHT DRIVING: THE ROLES OF ROD AND CONE PHOTORECEPTORS
}

\author{
Michael J. Flannagan \\ The University of Michigan \\ Transportation Research Institute \\ Ann Arbor, Michigan, USA \\ E-mail:mjf@umich.edu
}

\begin{abstract}
Summary: Because of the low light levels that are important in night driving, it is possible that both rods and cones contribute to visual performance for drivers at night. However, little evidence has been available about the relative contributions of these classes of photoreceptors. Partly, this may be because until recently the range of light sources has been limited, and consequently the question was not of great practical importance, at least for photometry. However, there is now a much larger variety of sources in use for vehicle lighting, including high-intensity discharge (HID) and light emitting diode (LED) sources. The greater variety of sources and spectral power distributions has increased interest in how the visual response of a driver's eyes at night varies with wavelength. New evidence indicates that the relative contributions of rods and cones may be different for different driving tasks or different aspects of a driver's visual experience at night: detection of pedestrians - the task that is most critically affected by darknessmay depend primarily on cones, while subjective impressions of brightness, and perhaps the conspicuity of emergency signals in the visual periphery, may be strongly influenced by rods.
\end{abstract}

\section{INTRODUCTION}

The response of the human eye varies with the wavelength of the radiation striking it, and the most effective range of wavelengths is what we call light. The two best-established ways of defining light are illustrated in Figure 1. The solid curve is the photopic luminous efficiency function. It is intended to describe the response of the eye at relatively high (daytime) light levels, and it reflects the combined contributions of the three types of cone photoreceptors in the retina. The dashed curve is the scotopic luminous efficiency function. It is intended to describe the response of the eye at relatively low (nighttime) light levels, and it reflects the contribution of the rod photoreceptors. The difference in peak sensitivity (about $50 \mathrm{~nm}$ ) is reasonably large relative to the whole range of sensitivity of either function, and the consequences for how light is measured (in either photopic or scotopic units) can be substantial.

A significant amount of driving in done at night, and the possibility that scotopic vision contributes to driving performance has been suggested for many years (e.g., Allen, 1970). Many of the light levels involved in night driving are in a range where rods, cones, or some mixture of rods and cones might contribute to visual performance (e.g., Plainis, Murray, \& Charman, 2005). However, the night driving environment is not homogeneous; it almost always involves an enormous range of luminance values, usually including a highly luminous area just in front of the 


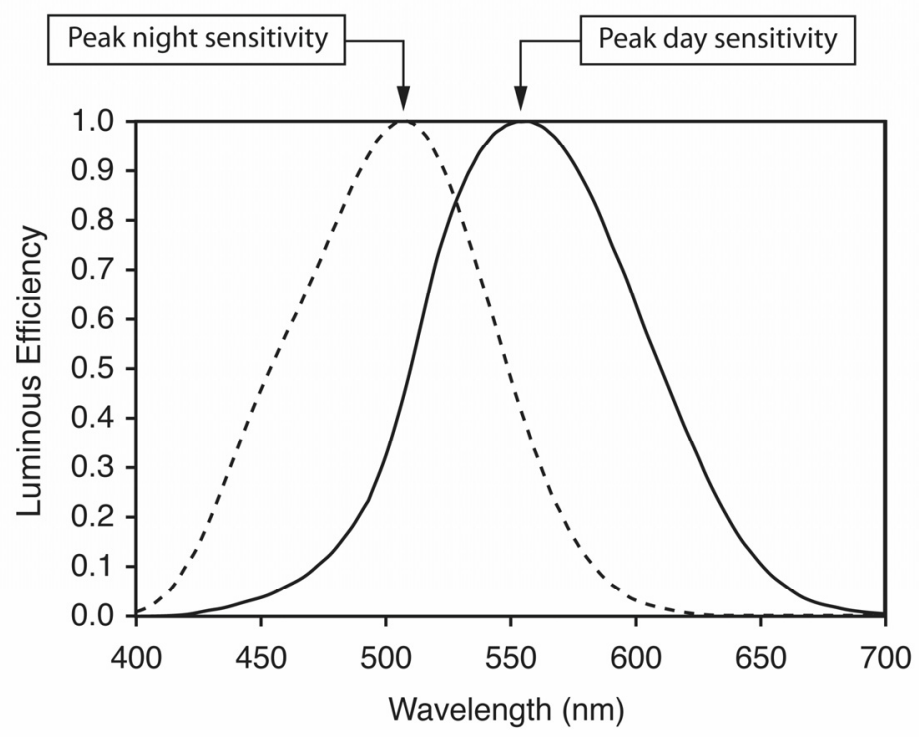

\section{Figure 1. The photopic (day, solid line) and scotopic (night, dashed line) luminous efficiency functions}

headlamps and peripheral areas of virtually complete darkness. Given the varied and changing lighting conditions and the often complex eye movement patterns that drivers exhibit, the nature of visual adaptation in night driving, and the relative contributions of rods and cones to overall visual performance, have not been fully analyzed. In addition to the inherent complexity of the problem, it may be that a lack of motivation has inhibited progress. Until recently, the spectra of light sources have been limited to the small range that can be produced by filament lamps. For example, in headlighting, tungsten bulbs were largely replaced by tungsten-halogen bulbs several decades ago (Moore, 1998), but the difference in spectra between these sources is small because they are both approximations of blackbody radiators. In contrast, automotive lighting now encompasses a much wider range of sources and spectra. Figure 2 illustrates the spectra of three light sources that are now in use to produce the nominally white light of headlamps, including tungsten-halogen filament bulbs, high-intensity discharge (HID) bulbs, and light-emitting diodes (LED). Figure 3 shows an even wider range of spectra: the red and blue stimuli produced by LED sources used in emergency vehicle warning lamps.

Although colored filters have long been used to produce strongly colored stimuli (for example, red stop lamps), the practical choices faced by lamp makers have limited the importance of fully understanding the effects of color. For example, in the U.S., vehicle manufacturers can produce vehicles with either red or yellow rear turn signals, and the U.S. federal standard for automotive lighting and related equipment (FMVSS 108, 2006) sets different minimum luminous intensity requirements for red and yellow rear turn signals: red rear turn signals must meet a minimum of 80 candelas, whereas yellow rear turn signals must meet a minimum of 130 candelas under the same conditions - a difference of over $60 \%$ in photometric values for lamps with identical functions. However, the difference in photometric values is not based on evidence about human visual performance, but on the practical consideration of how much light from a filament source will normally be passed by red and yellow filters. Now that LED lamps are being used to produce red and yellow stimuli without filtering, the old answer may be overly simplistic. 


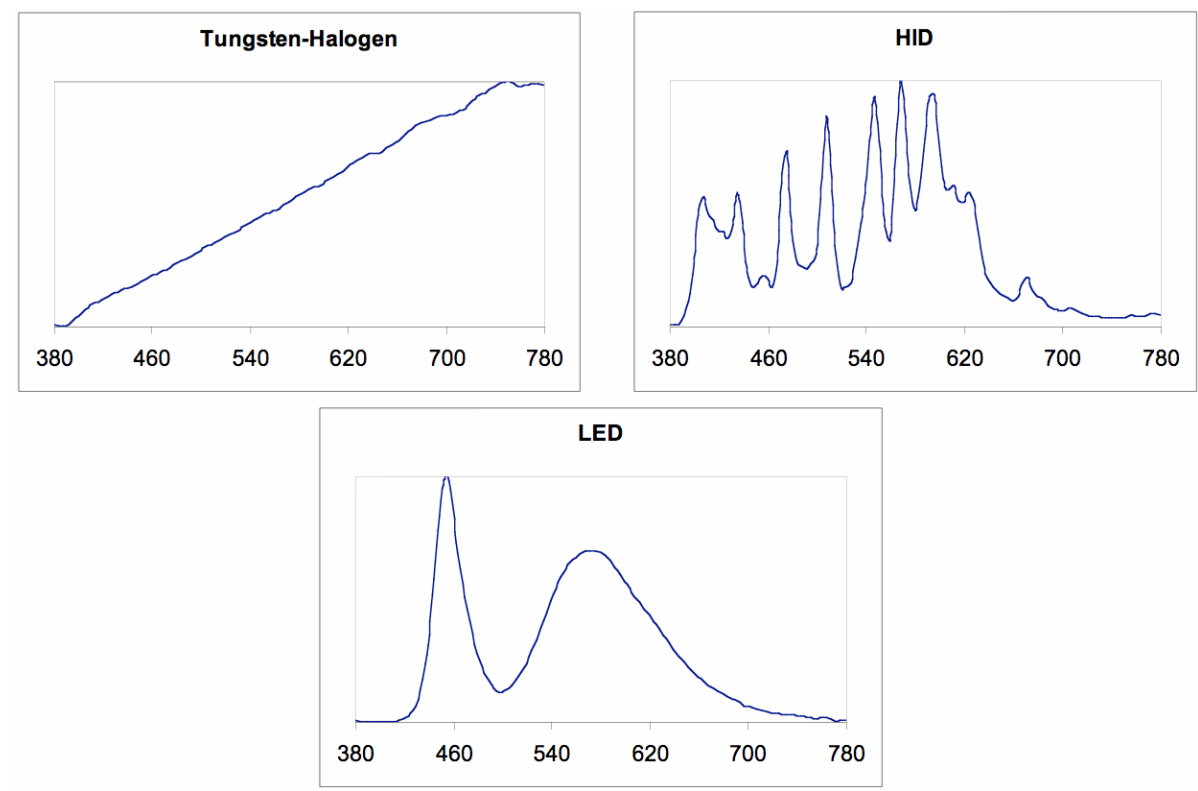

Figure 2. Spectra of various white light sources used in headlamps, as functions of wavelength $(\mathrm{nm})$

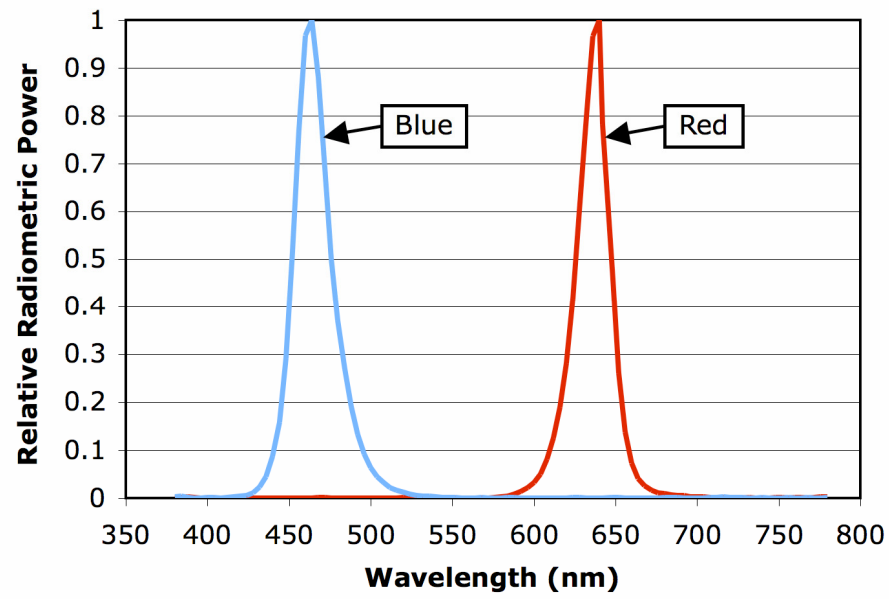

Figure 3. Spectra of blue and red LED emergency vehicle warning lamps

A preliminary assessment of the importance of the distinction between photopic and scotopic photometry for a given set of stimuli can be made by applying the functions in Figure 1 to spectra such as those in Figures 2 and 3. The result is often expressed as a scotopic/photopic, or $\mathrm{S} / \mathrm{P}$, ratio. This can be thought of as the number of photometric units that one would obtain by applying scotopic photometry to a given stimulus for every photometric unit that is measured by photopic photometry. (Actually, this is done using a slightly different pair of functions - the luminous efficacy functions - in which the scotopic function has a much higher peak than the photopic function, reflecting the fact that the rod system is much more sensitive than the cone system. The efficiency functions shown in Figure 1 graphically emphasize the spectral shift involved.) Table 1 shows the $\mathrm{S} / \mathrm{P}$ ratios for the three nominally white headlamp sources shown in Figure 2. The $\mathrm{S} / \mathrm{P}$ ratios differ by as much as $15 \%$, which is not a large range relative to the just 
noticeable difference estimate of $25 \%$ that has been recommended as a practical value for use with certain aspects of headlamps (Sayer, Flannagan, Sivak, Kojima, \& Flannagan, 1997).

Table 1. Chromaticity and $S / P$ ratio for each source shown in Figure 2

\begin{tabular}{|c|c|c|c|}
\hline \multirow{2}{*}{ Color } & \multicolumn{2}{|c|}{ CIE 1931 chromaticity } & \multirow{2}{*}{ S/P ratio } \\
\cline { 2 - 4 } & $\mathrm{x}$ & $\mathrm{y}$ & 1.50 \\
\hline \hline Tungsten-halogen & 0.434 & 0.405 & 1.70 \\
\hline HID & 0.377 & 0.387 & 1.72 \\
\hline LED & 0.353 & 0.340 & \\
\hline
\end{tabular}

Table 2 shows $\mathrm{S} / \mathrm{P}$ ratios for the LED sources illustrated in Figure 3. Here, the range is very large, indicating that the choice between photopic and scotopic photometry will be of great consequence, and therefore implying that it could be very important to determine the relative contributions of rods and cones to the visual tasks that the LED stimuli are meant to serve.

Table 2. Chromaticity and S/P ratio for the red and blue LEDs shown in Figure 3

\begin{tabular}{|c|c|c|c|}
\hline \multirow{2}{*}{ Color } & \multicolumn{2}{|c|}{ CIE 1931 chromaticity } & \multirow{2}{*}{ S/P ratio } \\
\cline { 2 - 4 } & $\mathrm{x}$ & $\mathrm{y}$ & 0.053 \\
\hline \hline Red LED & 0.701 & 0.297 & 16.1 \\
\hline Blue LED & 0.135 & 0.050 & \\
\hline
\end{tabular}

\section{EVIDENCE ABOUT PHOTORECEPTOR CONTRIBUTIONS}

Based on the foregoing discussion of S/P ratios, it might be expected that the choice between photopic and scotopic photometry for headlamps will not be of major importance, and that visual performance with lamps within the range roughly represented in Figure 2 will not vary greatly. That does indeed appear to be the case (Sullivan \& Flannagan, 2001). However, as indicated by the $\mathrm{S} / \mathrm{P}$ ratios in Table 2, there is potential for major differences in rod and cone contributions to the visual effectiveness of strongly colored lamps - the kind of lamps that are intended more for signaling and marking purposes than for road illumination. For example, the relative visual effectiveness of red and blue warning lamps on emergency vehicles may be very different in day versus night conditions if cones dominate daytime performance while rods make a substantial contribution at night.

Another domain in which the distinction between photopic and scotopic photometry may be important is vehicle interior lighting. Vehicle manufacturers have been developing more elaborate interior lighting systems for a variety of esthetic and functional purposes, and many of these systems involve colored light. We recently conducted a study intended to provide information about the visual effects of colored interior lighting (Devonshire \& Flannagan, 2007), and the main finding with regard to the relative contributions of rods and cones was that the answer was different for the two different visual tasks that we asked the participants to perform. The participants sat in a stationary car on a straight, dark test road at night. The car had its low- 
beam headlamps on, and we had set up a veiling reflection that covered most of the windshield from the participants' point of view (see Figure 4). The veiling reflection was produced by an LCD screen that was placed horizontally on the dashboard in front of the driver's position. It was meant to represent the kind of reflections that might be produced by a variety of interior light sources. The screen had been calibrated to produce a range of luminance values (equated in photopic $\mathrm{cd} / \mathrm{m}^{2}$ ) and colors (red, white, and blue; see Figure 5 and Table 3). The two tasks that the participants performed were (1) detection of a pedestrian walking toward the vehicle and away from it on the road ahead, and (2) magnitude estimation of the brightness of the veiling reflections. Our primary dependent variables were the distance at which the pedestrian was just detectable, and the log of the brightness magnitude estimates (using the log transformation to normalize over differences in range across participants). We chose the pedestrian detection task because pedestrian crashes appear to be the most critical safety issue for visibility in the darkness of night (Sullivan \& Flannagan, 2002).

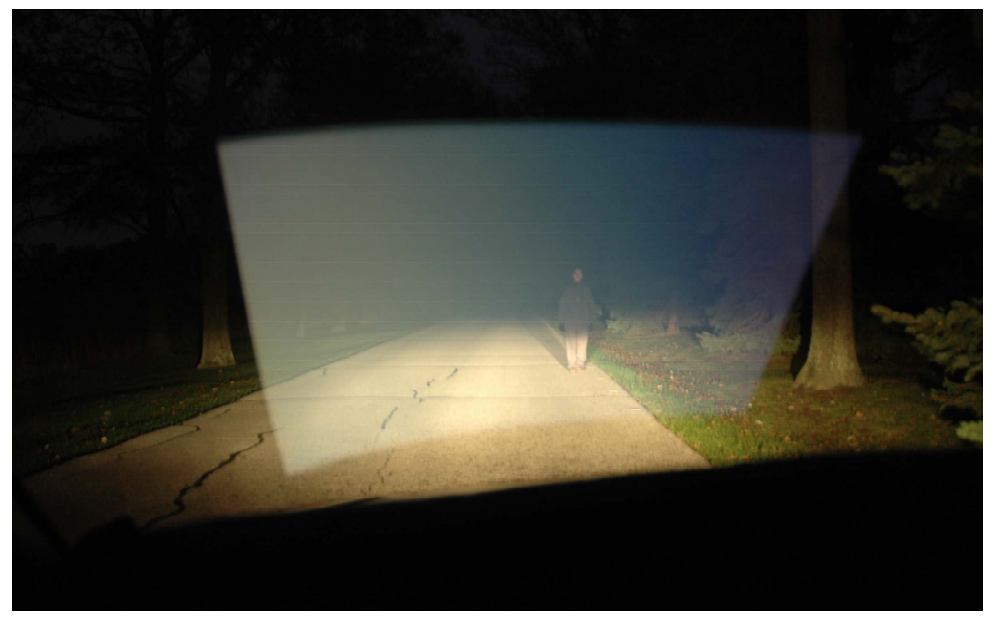

Figure 4. Participant's view of the pedestrian detection task

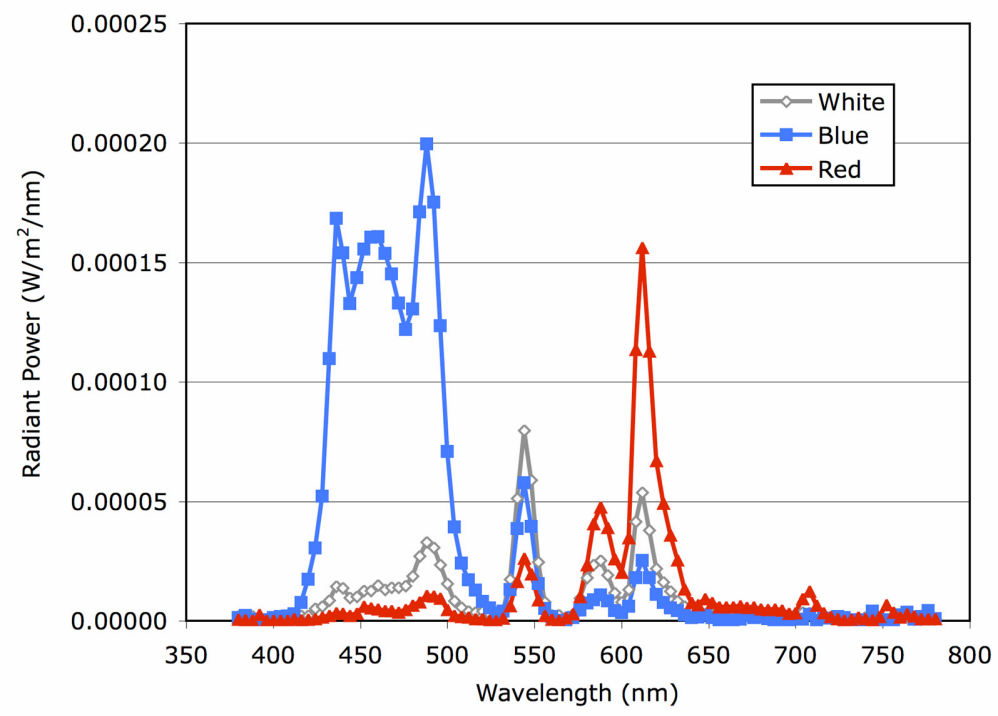

Figure 5. Spectra of the red, white, and blue veiling stimuli 
Table 3. Chromaticity and $S / P$ ratio for each of the veiling stimuli shown in Figure 5

\begin{tabular}{|c|c|c|c|}
\hline \multirow{2}{*}{ Color } & \multicolumn{2}{|c|}{ CIE 1931 chromaticity } & \multirow{2}{*}{ S/P ratio } \\
\cline { 2 - 3 } & $\mathrm{x}$ & $\mathrm{y}$ & 2.02 \\
\hline \hline White & 0.357 & 0.381 & 7.94 \\
\hline Blue & 0.163 & 0.125 & 0.69 \\
\hline Red & 0.564 & 0.364 & \\
\hline
\end{tabular}

Results for the pedestrian detection task and the brightness rating task are shown in the two panels of Figure 6. Detection distances decreased with greater veiling luminance, but there was no significant difference among the colors: detection distance for these markedly different colors was reasonably well predicted by the photopic units that we used to equate the stimuli. In contrast, for brightness ratings, there was a significant and substantial effect of color, with the blue stimuli receiving higher brightness ratings than either the white or red stimuli at equal photopic luminance levels. This result appears to indicate a contribution of rod photoreceptors to the brightness rating task, although there is no evidence of a rod contribution to the pedestrian detection task.
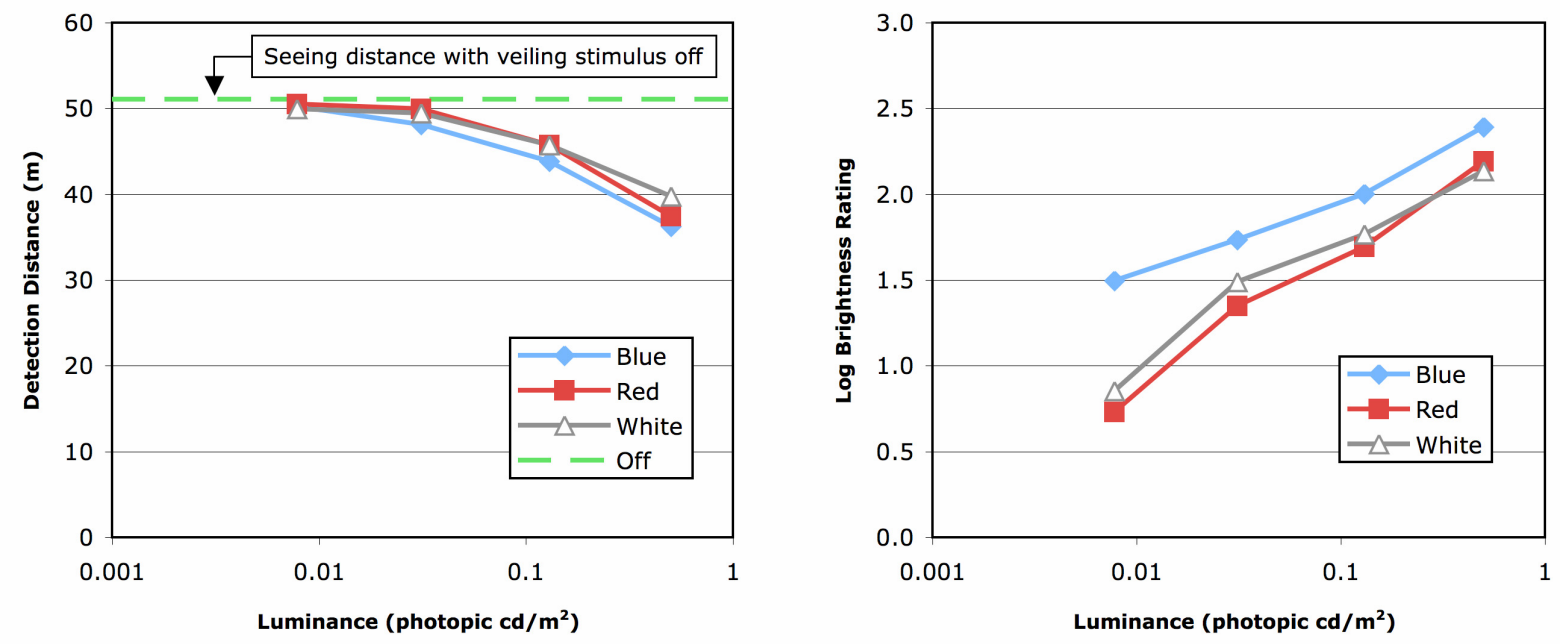

Figure 6. Pedestrian detection distances and log brightness ratings with various colors and luminance levels of veiling light

\section{SUMMARY AND CONCLUSIONS}

There has been little evidence that rod photoreceptors play an important role in driver vision at night. A belief that rods do not play an important role is most clearly embodied in the common practice of writing lighting standards in terms of photopic photometric units (e.g., FMVSS 108). The relative roles of rods and cones in night driving are still unclear, but recent developments in automotive light sources have made the issue more important than in the past, and there are some indications that rods do play a role in at least some aspects of drivers' visual experiences, and 
perhaps in the performance of some tasks. It appears that even under a single light adaptation condition, tasks that are apparently closely related may nevertheless differ in the relative contributions they involve from rods and cones. It may be that detection of pedestrians - the task that is most critically affected by darkness - depends primarily on cones, while subjective impressions of brightness, and perhaps the conspicuity of emergency signals in the visual periphery, may by strongly influenced by rods. Further work may be able to outline the characteristics of visual tasks that determine how they are served by these two major classes of photoreceptors.

\section{ACKNOWLEDGMENTS}

This work was supported by the University of Michigan Industry Affiliation Program for Human Factors in Transportation Safety. The current members are Alps Automotive/Alpine Electronics, Autoliv, Avery Dennison, Bendix, BMW, Bosch, Com-Corp Industries, DaimlerChrysler, Decoma Autosystems, Denso, Federal-Mogul, Ford, GE, General Motors, Gentex, Grote Industries, Hella, Honda, Ichikoh Industries, Koito Manufacturing, Lang-Mekra North America, Magna Donnelly, Muth, Nissan, North American Lighting, Northrop Grumman, OSRAM Sylvania, Philips Lighting, Renault, Sisecam, SL Corporation, Stanley Electric, Toyota Technical Center, USA, Truck-Lite, Valeo, Visteon/ACH, and 3M Personal Safety Products.

\section{REFERENCES}

Allen, M. J. (1970). Vision and highway safety. Philadelphia: Chilton.

Devonshire, J. M., \& Flannagan, M. J. (2007). Effects of automotive interior lighting on driver vision (Report No. UMTRI-2007-1). Ann Arbor: The University of Michigan Transportation Research Institute.

FMVSS 108. (2006). Federal Motor Vehicle Safety Standard 108: Lamps, reflective devices, and associated equipment, 49 C.F.R. $\$ 571.108$

Moore, D. (1998). Headlamp history and harmonization (Report No. UMTRI-98-21). Ann Arbor: The University of Michigan Transportation Research Institute.

Plainis, S., Murray, I. J., \& Charman, W. N. (2005). The role of retinal adaptation in night driving. Optometry and Vision Science, 82(8), 682-688.

Sayer, J. R., Flannagan, M. J., Sivak, M., Kojima, S., \& Flannagan, C. C. (1997). Just noticeable differences for low-beam headlamp intensities (Report No. UMTRI-97-4). Ann Arbor: The University of Michigan Transportation Research Institute.

Sullivan, J. M., \& Flannagan, M. J. (2001). Visual effects of blue-tinted tungsten-halogen headlamp bulbs (Report No. UMTRI-2001-9). Ann Arbor: The University of Michigan Transportation Research Institute.

Sullivan, J. M., \& Flannagan, M. J. (2002). The role of ambient light level in fatal crashes: inferences from daylight saving time transitions. Accident Analysis and Prevention, 34, 487498. 